\title{
THE PROBABILITY GENERATING FUNCTIONAL
}

\author{
M. WESTCOTT
}

(Received 22 June 1970)

Communicated by P. D. Finch

\section{Introduction}

This paper is concerned with certain aspects of the theory and application of the probability generating functional (p.g.fl) of a point process on the real line. Interest in point processes has increased rapidly during the last decade and a number of different approaches to the subject have been expounded (see for example [6], [11], [15], [17], [20], [25], [27], [28]). It is hoped that the present development using the p.g.fl will clarify and unite some of these viewpoints and provide a useful tool for the solution of particular problems.

Because of the variety of papers on the theory of point processes (indeed, the whole subject has a very scattered literature) we find it necessary to collect the relevant parts of the several approaches in a preparatory section on point processes. Then follows the definition of the p.g.fl, with some examples and properties. Most of these are known in the case of population processes (Moyal [20] and Harris [6]) but are new in this generality, though the extension is usually not difficult. Section 4 deals with a characterisation of the p.g.fl among one class of functionals and gives an example. In Section 5 we develop a Taylor expansion of the p.g.fl in terms of moment measures of the associated point process and show how it may facilitate the proofs of limit theorems. Section 6 establishes a characterisation of mixing and ergodic point processes in terms of their p.g.fls, in analogy with some results of Leonov [14], followed by several examples of such processes. We conclude with a few additional remarks.

\section{Point processes}

In this section we mention some basic results for point processes (or random streams) which will be needed subsequently.

Let $\Omega$ be the set of all countable sequences of real numbers $\left\{t_{i}\right\}$ without limit points and let $N(A)$ be the cardinality of the set $\left\{t_{i} \in A\right\}$ for all Borel sets $A$ on the real line. Then $N(\cdot)$ is a counting measure i.e. a non-negative integer valued 
set function countably additive on the Borel sets. Since $\left\{t_{i}\right\}$ has no limit points $N(\cdot)$ is obviously finite on bounded sets. It is known (Moyal [20]) that there is a one-to-one correspondence between $\Omega$ and the set of all $\sigma$-finite counting measures $N(\cdot)$, which we therefore also denote by $\Omega$.

Consider now a set of functions $p\left(A_{1}, \cdots, A_{k} ; r_{1}, \cdots, r_{k}\right)$ where $k, r_{1}, \cdots, r_{k}$ are non-negative integers and $A_{1}, \cdots, A_{k}$ are Borel sets. In order that they be the finite-dimensional distributions of a point process they must satisfy the following consistency conditions:

(1) $p\left(A_{1}, \cdots, A_{k} ; r_{1}, \cdots, r_{k}\right)=p\left(A_{i_{1}}, \cdots, A_{i_{k}} ; r_{i_{1}}, \cdots, r_{i_{k}}\right)$ for any permutation $\left(i_{1}, \cdots, i_{k}\right)$ of $(1, \cdots, k)$;

$$
\begin{aligned}
& p\left(A_{1}, \cdots, A_{k} ; r_{1}, \cdots, r_{k}\right) \geqq 0 \text { and } \sum_{r=0}^{\infty} p\left(A_{1}, \cdots, A_{k}, A ; r_{1}, \cdots, r_{k}, r\right) \\
& =p\left(A_{1}, \cdots, A_{k} ; r_{1}, \cdots, r_{k}\right) ;
\end{aligned}
$$

(3) $p\left(A_{1} \cup \cdots \cup A_{k} ; r\right)=\Sigma_{r_{1}+\cdots+r_{k}=r} p\left(A_{1}, \cdots, A_{k} ; r_{1}, \cdots, r_{k}\right)$ when the $A_{i}$ are disjoint;

(4) If a sequence of bounded sets $A_{k} \downarrow \varnothing$, the null set, as $k \rightarrow \infty$ then

$$
\lim _{k \rightarrow \infty} p\left(A_{k} ; 0\right)=1 \text {. }
$$

The fundamental result is

THEOREM 1. (Moyal [20], Harris [6] p. 55, Nawrotzki [22]). Corresponding to a set of functions $p\left(A_{1}, \cdots, A_{k} ; r_{1}, \cdots, r_{k}\right)$ satisfying (1)-(4) there is a unique probability measure $\mathscr{P}$ defined on the $\sigma$-algebra $\mathscr{F}$ generated by the cylinder sets $\left\{N(\cdot) ; N\left(A_{1}\right)=r_{1}, \cdots, N\left(A_{k}\right)=r_{k}\right\}$ for which

$$
\mathscr{P}\left\{N\left(A_{1}\right)=r_{1}, \cdots, N\left(A_{k}\right)=r_{k}\right\}=p\left(A_{1}, \cdots, A_{k} ; r_{1}, \cdots, r_{k}\right) .
$$

A point process is specified by the triple $(\Omega, \mathscr{F}, \mathscr{P})$. Since the Borel sets on the real line can be generated by half-open intervals we may consider the $p(\cdot ; \cdot)$ of Theorem 1 only for disjoint half-open intervals. This is sometimes convenient. Notationally we will write $N(\cdot)$ for an arbitrary point process on the real line (for generalisations see [20]), assuming always it is finite on bounded Borel sets.

For an alternative approach to the definition of a point process we refer to Ryll-Nardzewski [25] and Matthes [15], who take $\mathscr{F}$ as the smallest $\sigma$-algebra for which $N(A)$ is a random variable for all Borel sets $A$. The equivalence of the two outlooks is proved in [15] or [20].

A point process $N(\cdot)$ is stationary if

$$
p\left(T^{t} A_{1}, \cdots, T^{t} A_{k} ; r_{1}, \cdots, r_{k}\right)=p\left(A_{1}, \cdots, A_{k} ; r_{1}, \cdots, r_{k}\right),
$$


where the translation operator $T$ is defined by $T^{t} A=\{x: x+t \in A\}$. We see that if $N(\cdot)$ is stationary, $N(-\infty, \infty)=0$ or $\infty$ with probability one.

A stationary point process is ergodic if all events invariant under translation have probability zero or one. A necessary and sufficient condition for ergodicity is (c.f. Rosenblatt [23] p. 110)

$$
\lim _{t \rightarrow \infty} t^{-1} \int_{0}^{t} \mathscr{P}\left(A \cap T^{-\tau} B\right) d \tau=\mathscr{P}(A) \mathscr{P}(B) \quad \text { for all } A, B \in \mathscr{F} .
$$

The process is mixing if

$$
\lim _{t \rightarrow \infty} \mathscr{P}\left(A \cap T^{-t} B\right)=\mathscr{P}(A) \mathscr{P}(B) \quad \text { for all } A, B \in \mathscr{F},
$$

and is weakly mixing if

$$
\lim _{t \rightarrow \infty} t^{-1} \int_{0}^{t}\left|\mathscr{P}\left(A \cap T^{-\tau} B\right)-\mathscr{P}(A) \mathscr{P}(B)\right| d \tau=0 \quad \text { for all } A, B \in \mathscr{F} .
$$

Obviously mixing $\Rightarrow$ weak mixing $\Rightarrow$ ergodic (Matthes [15]).

The superposition of $n$ independent point processes $N_{1}(\cdot), \cdots, N_{n}(\cdot)$ is simply the aggregation of all their points, written $\sum_{i=1}^{n} N_{i}(\cdot)$, and has finitedimensional distributions derived by convolution as usual.

A sequence $\left\{N_{n}(\cdot)\right\}$ of point processes converges in distribution to a point process $N(\cdot)$, in symbols $N_{n}(\cdot) \stackrel{d}{\rightarrow} N(\cdot)$, if all the finite-dimensional distributions converge in the usual sense. For a stronger concept see Jiřina [8].

We can define integrals with respect to a point process by

$$
\int f(t) d N(t)=\sum_{i} f\left(t_{i}\right)
$$

where $\int$ always means $\int_{-\infty}^{\infty}$, for such functions $f$ as ensure existence. Obviously the class of functions which vanish outside some bounded interval make the integral finite and other criteria are considered later.

The moment structure of a point process is determined by a set of moment measures, namely expectations of product counting measures on $\Omega \times \cdots \times \Omega$. The first moment measure $M(\cdot)=E\{N(\cdot)\}$ is always assumed to be a Borel measure (Halmos [5] p. 223). Thus $M(A)<\infty$ for any bounded set $A$ which implies $N(A)<\infty$ with probability one, an assumption already made. Clearly for a stationary process $M(A)=m|A|$, where $|A|$ is the Lebesgue measure of $A$ and $m=E\{N[0,1)\}$ is the intensity of $N(\cdot)$.

For higher moment measures like $M_{(2)}(A \times B)=E\{N(A) N(B)\}$ we find concentrations on subsets of lower dimension. Consequently we prefer to employ the factorial moment measures $M_{n}(\cdot)$ defined by analogy with the factorial moments (see Moyal [20], Vere-Jones [28], though their notation is reversed here). For example 


$$
M_{2}(A \times B)=E\{N(A) N(B)\}-E\{N(A \cap B)\},
$$

which equals $M_{(2)}(\cdot)$ if $A \cap B=\varnothing$ and the usual factorial moment if $A=B$. Under reasonable conditions the diagonal concentrations now disappear.

We can also define factorial cumulant measures $C_{n}(\cdot)$ by the usual momentcumulant formulae. If these measures are absolutely continuous we may speak of factorial moment and cumulant densities $m_{n}(\cdot)$ and $c_{n}(\cdot)$, which are the product densities of Bartlett [2] pp. 83, 122.

Fubini's theorem now shows that for integrals (4)

$$
E\left\{\int f(t) d N(t)\right\}=\int f(t) M(d t)
$$

if $\int|f(t)| M(d t)<\infty$, in which case the integral exists. Similar considerations apply to higher moments and when $f$ is an indicator function we get the moments of $N[0, t)$ in terms of moment measures (Vere-Jones [28], [29]; Cox and Lewis [3] Ch. 4).

The most important point process is of course the Poisson process, whose fundamental property is having independent increments (Khintchine [11] $\S 3$ ). In general its parameter is a measure $\Lambda(\cdot)$, when we speak of a Poisson $(\Lambda(\cdot))$ process, but under further assumptions this becomes a function $\lambda(t) \circ$ even a constant $\lambda([11], \S \S 3,5,8)$. We are particularly interested in the case of a random parameter, which when constant gives a mixed Poisson process and when a function of $t$ produces a doubly stochastic Poisson process (Cox and Lewis [3] p. 179). An important special case is a linear mean process, when we get the linear stochastic Poisson process of Westcott [30].

Also of interest are the infinitely divisible point processes introduced by Matthes [16] and Lee [12] and studied in Kerstan and Matthes [9], [10], Lee [13]. There are a number of equivalent definitions of such processes, for which see [16]. One consequence of them is that all the finite-dimensional distributions are infinitely divisible in the usual sense and hence are compound Poisson with probability generating functions (p.g.fs) of the form $\exp \left(\Sigma_{m} a(m) z^{m}\right)$. In fact we have

Lemma 1 ([9], [12]). To each infinitely divisible point process $(\Omega, \mathscr{F}, \mathscr{P})$ there corresponds exactly one measure $\tilde{\mathscr{P}}(\cdot)$ on $\mathscr{F}$ with the properties

(i) $\tilde{\mathscr{P}}\left\{N\left(I_{1}\right)=r_{1}, \cdots, N\left(I_{k}\right)=r_{k}\right\}=a\left(r_{1}, \cdots, r_{k} ; I_{1}, \cdots, I_{k}\right)$ for all integers $k$, $r_{1}, \cdots, r_{k}$ and intervals $I_{1}, \cdots, I_{k}$;

(ii) $\tilde{\mathscr{P}}\{\varnothing\}=0$;

(iii) $\tilde{\mathscr{P}}\{N(I) \neq 0\}<\infty$ for all bounded intervals $I$.

The KLM (Kerstan-Lee-Matthes) measure $\tilde{\mathscr{P}}$ is stationary if and only if $\mathscr{P}$ is.

We may now define regular and singular infinitely divisible point processes 
(Matthes [16]), corresponding to $\tilde{\mathscr{P}}$ concentrated on members of $\Omega$ having finitely and infinitely many points respectively. For examples of KLM measures see Lee [13].

\section{The probability generating functional}

We consider throughout a point process $N(\cdot)$ whose expectation measure is a Borel measure. It may or may not be almost surely finite.

DEFINITION. The probability generating functional (p.g.f) of $N(\cdot)$ is defined by

$$
G[\xi]=E\left\{\exp \int \log \xi(t) d N(t)\right\}
$$

for a suitable class of functions $\xi$. Equivalently

$$
G[\xi]=E\left\{\prod_{i} \xi\left(t_{i}\right)\right\},
$$

where the $\left\{t_{i}\right\}$ are the positions of the points; $\left\{t_{i}\right\} \in \Omega$.

In this generality the definitions are due to Vere-Jones [28]. For the special case of almost surely finite numbers of points see Moyal [20].

It is clear that heuristically the p.g.fl is an extension of the multivariate p.g.f. to the 'generating function' of an infinite set of 'random variables' $d N(t)$. We expect then that its properties will be similar to those of the p.g.f. and later we shall see this is generally true.

To ensure that the p.g.fl. is nontrivial the exponent in (5) must be finite with probability one. This motivates the next

Definition. If $\xi$ is measurable and $0 \leqq \xi(t) \leqq 1$ for all real $t$ then $\xi \in V$ if $\xi$ vanishes outside a bounded interval and $\xi \in L(N)$ if $\int|\log \xi(t)| M(d t)<\infty$, where $M(\cdot)=E\{N(\cdot)\}$.

These are the classes introduced by Vere-Jones [28]. We must further decide what happens in (5) at zeros of $\xi(t)$. If $\xi(t) \equiv 0$ over some set $A$ the exponential in (5) is taken as zero, unless $N(A)=0$ when it equals one. Now since $M(\cdot)$ is a Borel measure we have ([28])

LeMma 2. $G[\xi]$ is non-trivial if

(i) $N(\cdot)$ is almost surely finite

or (ii) $1-\xi \in V$

or (iii) $\xi \in L(N)$; in this case $\int[1-\xi(t)] M(d t)<\infty$ also.

If in future we use a p.g.fl without specific reference to $\xi$ we are assuming that it belongs to either of the above classes.

The next lemma shows the fundamental role that the p.g.fl plays in the theory of point processes. 
Lemma 3. ([20], [28]). The p.g. $f$ is uniquely determined by $N(\cdot)$ and, conversely, knowledge of the p.g. $A$ completely determines the probability structure of $N(\cdot)$

An important consequence is that the p.g.fs of the finite-dimensional distributions of $N(\cdot)$ are derived from the p.g.fl by setting $\xi$ equal to a simple function.

In view of Lemma 3 the p.g.fl is obviously a powerful aid in the study of point processes, containing, as it does, information about all aspects of the process. Its disadvantage however is that it is rarely obtainable in closed form unless the point process involved is related to the Poisson process. Some examples of this appear below. Nevertheless it is a valuable tool in a variety of theoretical problems such as characterisations of ergodic and mixing point processes (Section 6), the theory of cluster processes and limit theorems for point processes.

For a Poisson $(\Lambda(\cdot))$ process the completely random property easily gives

$$
G[\xi]=\exp \left\{-\int[1-\xi(t)] \Lambda(d t)\right\} ;
$$

c.f. Ryll-Nardzewski [24], Moyal [20], Shiryaev [26]. In particular, for the stationary Poisson $(\lambda)$ process

$$
G[\xi]=\exp \left\{-\lambda \int^{\infty}[1-\xi(t)] d t\right\} .
$$

The p.g.fl of a doubly stochastic Poisson process with a stationary mean process $\Lambda(t)$ is readily obtained from (7); first, however, we need the

Definition. The Laplace functional of a non-negative stochastic process $Y(t)$ is

$$
L_{Y}[\xi]=E\left\{e^{-\int Y(t) \xi(d t)}\right\},
$$

where $\xi(\cdot)$ is a totally finite measure on the Borel sets of the real line.

This definition is based on Shiryaev [26]; for a related idea see Jiřina [8]. Clearly, a sufficient condition for (9) to be nontrivial is that $Y(t)$ be stationary with finite mean, in which case a conditional argument due to Bartlett [1] gives the desired p.g.fl as

$$
G[\xi]=L_{\Lambda}\left[\int_{-\infty}^{t}[1-\xi(u)] d u\right] .
$$

When $\Lambda(t)$ is linear, producing a linear stochastic Poisson process, (10) may be evaluated more explicitly as in Westcott [30].

We now develop further properties of the p.g.fl. Obviously

(a) $0 \leqq G[\xi] \leqq 1$ and

(b) $G$ is monotonic i.e. $\xi_{1} \leqq \xi_{2} \Rightarrow G\left[\xi_{1}\right] \leqq G\left[\xi_{2}\right]$. 
(c) We might assume that the p.g.fl is always continuous, in the sense that $\xi_{n}(t) \rightarrow \xi(t)$ pointwise as $n \rightarrow \infty$ implies $G\left[\xi_{n}\right] \rightarrow G[\xi]$. To see this is in general false consider $\xi_{n}(t)=1-(1-z) \chi_{[n, n+1)}(t)$, where $\chi_{A}$ is the indicator function of a set $A$, so that $G\left[\xi_{n}\right]=E\left\{z^{N[n, n+1)}\right\}$. Clearly $\xi_{n}(t) \rightarrow 1$ pointwise as $n \rightarrow \infty$ yet $G\left[\xi_{n}\right\}$ need not tend to 1 (take $N(\cdot)$ stationary for example). However we do have

THEOREM 2. The p.g.f is continuous if one of the following holds:

(i) $N(\cdot)$ is almost surely finite,

(ii) the $1-\xi_{n} \in V$ and have a common interval outside which they all vanish,

(iii) $\xi_{n}(t) \geqq \tilde{\xi}(t)$ and $\tilde{\xi} \in L(N)$,

(iv) $\int\left|\xi_{n}(t)-\xi(t)\right| M(d t) \rightarrow 0$ as $n \rightarrow \infty$,

(v) $\xi \in L(N)$ and given $\varepsilon>0$ there is $T(\varepsilon)$ such that for all $n$

$$
\int_{|t|>T}\left|\log \xi_{n}(t)\right| M(d t)<\varepsilon \text { or } \int_{|t|>T}\left[1-\xi_{n}(t)\right] M(d t)<\varepsilon .
$$

Proof. In each case we prove that

$$
\int \log \xi_{n}(t) d N(t) \rightarrow \int \log \xi(t) d N(t)
$$

in some sense, as then the bounded convergence theorem ensures continuity. (i) is obvious (see [6] p. 58). The assumptions in (ii) effectively reduce it to (i) and (iii) follows directly from dominated convergence. To prove (iv) we use the simple identity, due to Moyal [21],

$$
\prod_{i=1}^{n} \zeta\left(t_{i}\right)-\prod_{i=1}^{n} \eta\left(t_{i}\right)=\sum_{i=1}^{n}\left[\zeta\left(t_{i}\right)-\eta\left(t_{i}\right)\right] \eta\left(t_{1}\right) \cdots \eta\left(t_{i-1}\right) \zeta\left(t_{i+1}\right) \cdots \zeta\left(t_{n}\right)
$$

valid for $n=1,2, \cdots$ and any functions $\zeta, \eta$. With $\zeta=\xi_{n}, \eta=\xi$ we see from (4) and (6) that

$$
\begin{aligned}
\left|G\left[\xi_{n}\right]-G[\xi]\right| & \leqq E\left\{\int\left|\xi_{n}(t)-\xi(t)\right| d N(t)\right\} \\
& =\int\left|\xi_{n}(t)-\xi(t)\right| M(d t) \rightarrow 0 \text { as } n \rightarrow \infty .
\end{aligned}
$$

As the conditions (i)-(iv) all ensure that $\xi$ satisfies Lemma 2 we can consistently write $G[\xi]$ for the limit. If now we assume $\xi \in L(N)$ then, taking $\int_{|t|<T}+\int_{|r|>T}$ and recalling $M(\cdot)$ is a Borel measure, (i) and (iv) can be used to establish (v).

(d) As we might expect, superposition of independent streams is equivalent to multiplication of p.g.fls. Thus if $N_{1}(\cdot), \cdots, N_{k}(\cdot)$ are independent point processes with p.g.fls $G_{1}[\xi], \cdots, G_{k}[\xi]$ the p.g.fl of $\sum_{i=1}^{k} N_{i}(\cdot)$ is

$$
G[\xi]=\prod_{i=1}^{k} G_{i}[\xi] \text {. }
$$

This is immediately apparent from (5) or (6). 
(e) A useful property of the p.g.f is that it provides necessary and sufficient conditions for the convergence of discrete probability measures. The analogous result for point processes is given by the following minor extension of Vere-Jones [28].

Lemma 4. A sequence of point processes $\left\{N_{n}(\cdot)\right\}$ converges in distribution to a point process $N(\cdot)$ if and only if the associated p.g. Als converge i.e. $G_{n}[\xi] \rightarrow G[\xi]$ as $n \rightarrow \infty$ for every $1-\xi \in V$.

Proof. The sufficiency of the condition is obvious. To prove necessity, we note that for any $\xi, 1-\xi \in V$, there are upper and lower sequences of simple functions $\xi_{m}, \xi_{m}^{\prime}$ approximating $\xi$. By (b)

$$
G_{n}\left[\xi_{m}^{\prime}\right] \leqq G_{n}[\xi] \leqq G_{n}\left[\xi_{m}\right]
$$

for all $m$ and $n$, and as $n \rightarrow \infty$

$$
G\left[\xi_{m}^{\prime}\right] \leqq \liminf _{n \rightarrow \infty} G_{n}[\xi] \leqq \limsup _{n \rightarrow \infty} G_{n}[\xi] \leqq G\left[\xi_{m}\right]
$$

from the definition of convergence in distribution. Then as $m \rightarrow \infty$ the outer terms converge to $G[\xi]$ by Theorem 2(ii) i.e.

$$
\lim _{n \rightarrow \infty} G_{n}[\xi]=G[\xi] .
$$

We note that a stronger assertion, corresponding to weak convergence of measures, is given for Laplace functionals by Jiřina [8]. Also, Lemma 4 can be generalised slightly if we assume only that the $G_{n}[\xi]$ converge to some functional $G[\xi]$. Specifically we have

THEOREM 3. A sequence of point processes $\left\{N_{n}(\cdot)\right\}$ converges in distribution to a point process $N(\cdot)$ if and only if the p.g.fls $G_{n}[\xi]$ converge to a functional $G[\xi]$ which is continuous for sequences $\xi_{m}$ satisfying Theorem 2(ii) and such that $\xi_{m}(t) \rightarrow 1$ pointwise. Then $G[\xi]$ is the p.g. $f$ of $N(\cdot)$.

ProOF. The 'only if' part follows as before. For the sufficiency, take a simple function $\xi(t)=1-\sum_{i=1}^{k}\left(1-z_{i}\right) \chi_{A_{i}}(t)$, when $G_{n}[\xi]=P_{n}\left(z_{1}, \cdots, z_{k} ; A_{1}, \cdots, A_{k}\right)$ becomes the p.g.f of some joint distribution of $N_{n}(\cdot)$ and converges to a function $G[\xi]=P\left(z_{1}, \cdots, z_{k}, A_{1}, \cdots, A_{k}\right)$ that is continuous as $\left(z_{1}, \cdots, z_{k}\right) \rightarrow(1, \cdots, 1)$. So by a standard result $G[\xi]$ is also a p.g.f and therefore all the joint p.g.fs of $N_{n}(\cdot)$ converge to a set of p.g.fs. We now prove that they are consistent, in the sense of satisfying (1)-(4). It is easy to see that

$$
\begin{aligned}
P\left(z_{1}, z_{2} ; A_{1}, A_{2}\right)=\lim _{n \rightarrow \infty} P_{n}\left(z_{1}, z_{2} ; A_{1}, A_{2}\right) & =\lim _{n \rightarrow \infty} P_{n}\left(z_{2}, z_{1} ; A_{2}, A_{1}\right) \\
& =P\left(z_{2}, z_{1} ; A_{2}, A_{1}\right)
\end{aligned}
$$

and so on, as the $P_{n}$ satisfy (1). 
(2)' $P_{n}\left(z_{1}, z_{2} ; A_{1}, A_{2}\right) \rightarrow P\left(z_{1}, z_{2} ; A_{1}, A_{2}\right)$ by hypothesis

and $P_{n}\left(z_{1}, 1 ; A_{1}, A_{2}\right)=P_{n}\left(z_{1} ; A_{1}\right)$ by (2),

i.e. $P\left(z_{1}, 1 ; A_{1}, A_{2}\right)=P\left(z_{1} ; A_{1}\right)$ and so on.

(3)' $P_{n}\left(z_{1}, z_{2}, z_{3} ; A_{1}, A_{2}, A_{1} \cup A_{2}\right) \rightarrow P\left(z_{1}, z_{2}, z_{3} ; A_{1}, A_{2}, A_{1} \cup A_{2}\right), A_{1} \cap A_{2}=\varnothing$

and $P_{n}\left(z_{1}, z_{2}, z_{3} ; A_{1}, A_{2}, A_{1} \cup A_{2}\right)=P_{n}\left(z_{1} z_{3}, z_{2} z_{3} ; A_{1}, A_{2}\right)$ by (3)

$$
\rightarrow P\left(z_{1} z_{3}, z_{2} z_{3} ; A_{1}, A_{2}\right) \text { and so on. }
$$

(4) Take a sequence of bounded sets $A_{m} \downarrow \varnothing$ and define $\xi_{m}(t)=1-(1-z) \chi_{A m}(t)$. Then $\xi_{m}(t) \rightarrow 1$ pointwise as $m \rightarrow \infty$ and so $G\left[\xi_{m}\right] \rightarrow G[1]=1$. But $G\left[\xi_{m}\right]$ $=P\left(z ; A_{m}\right)$.

So the limit distributions form a consistent set and by Theorem 1 there is a unique point process $N(\cdot)$ having them as its finite-dimensional distributions. If $N(\cdot)$ has p.g.fl $G^{*}[\xi]$ then the 'only if' part gives $G_{n}[\xi] \rightarrow G^{*}[\xi]$ whereas $G_{n}[\xi] \rightarrow G[\xi]$ by hypothesis. Thus $G[\xi]$ is the p.g.fl of $N(\cdot)$.

\section{A characterisation result for the p.g.ff.}

Our next problem is to characterise the functionals over $V$ which are p.g.fls. In the almost surely finite case Harris [6] p. 58 gives a result involving those functionals whose arguments are simple functions and Moyal [20] characterises p.g.fls in terms of restrictions to finite subsets of the population space. Our theorem is not essentially new and draws on this previous work.

The basic technique is due to Harris ([6] p. 53), namely that a set of functions $p_{0}\left(A_{1}, \cdots, A_{k} ; r_{1}, \cdots, r_{k}\right)$ satisfying (1)-(4) for disjoint sets $A_{i}$ (with slight changes in (3)) can be uniquely extended to functions $p\left(A_{1}, \cdots, A_{k} ; r_{1}, \cdots, r_{k}\right)$ satisfying (1)-(4) and agreeing with the $p_{0}(\cdot ;)$ whenever the $A_{i}$ are disjoint.

THEOREM 4. Suppose we have a functional $G[\xi]$ defined whenever $1-\xi \in V$ and continuous for sequences $\xi_{n}$ satisfying Theorem 2(ii). Further, if $1-\xi(t)$ $=\sum_{i=1}^{k}\left(1-z_{i}\right) \chi_{A_{i}}(t)$ where the Borel sets $A_{i}$ are disjoint, suppose $G[\xi]$ $=P\left(z_{1}, \cdots, z_{k} ; A_{1}, \cdots, A_{k}\right)$ is the p.g.f. of an $n$-dimensional discrete random variable. Then $G[\xi]$ is the p.g.fl of a point process.

Proof. Let the distribution associated with $P\left(z_{1}, \cdots, z_{k} ; A_{1}, \cdots, A_{k}\right)$ be $p_{0}\left(A_{1}, \cdots, A_{k} ; r_{1}, \cdots, r_{k}\right)$ for disjoint $A_{i}$. In the consistency conditions

(1) holds because $p_{0}(\cdot ; \cdot)$ is certainly a probability distribution,

(2) holds by the obvious relation $P\left(z_{1}, \cdots, z_{k}, 1 ; A_{1}, \cdots, A_{k}, A_{k+1}\right)$ $=P\left(z_{1}, \cdots, z_{k} ; A_{1}, \cdots, A_{k}\right)$,

(3) holds as clearly $P\left(z, z ; A_{1}, A_{2}\right)=P\left(z ; A_{i} \cup A_{2}\right)$ and this can be extended to the disjoint collections $\left\{A_{i j}\right\}$ making up the $A_{i}$ (for explanation see [6]),

(4) holds by the continuity of $G$ for functions of the form $\xi_{n}(t)=1-(1-z) \chi_{A}(t)$, bounded $A_{n} \downarrow \varnothing$, for which $\xi_{n} \rightarrow 1$ pointwise. 
So we may extend the $p_{0}(\cdot ; \cdot)$ uniquely to a consistent set of functions which by Theorem 1 are the finite-dimensional distributions of a unique point process $N(\cdot)$. Its p.g.fi $G^{*}[\xi], 1-\xi \in V$, must agree with $G[\xi]$ over simple functions $\xi$. But arbitrary $1-\xi \in V$ can be uniformly approximated by an increasing sequence of simple functions and $G, G^{*}$ are continuous for such sequences by hypothesis and Theorem 2(ii) respectively. Therefore they agree for all $\xi$, $1-\xi \in V$.

To illustrate the application of this theorem we determine the canonical form for the p.g.fl of an infinitely divisible point process with KLM measure $\tilde{P}$. Consider the functional

$$
\left.G[\xi]=\exp \int_{\Omega-\{\phi\}}\left[e^{\int \log \zeta(t) d N(t)}-1\right] \tilde{\mathscr{P}}(d N)\right\}
$$

for $1-\xi \in V$. For simple functions $\xi$ Lemma 1 shows that $G[\xi]$ becomes the p.g.f. of a compound Poisson distribution. Now suppose we have a sequence $1-\xi_{n} \in V$, all vanishing outside a common interval $I$, with $\xi_{n} \rightarrow \xi$ pointwise as $n \rightarrow \infty$. By (iii) of Lemma $1 \tilde{\mathscr{P}}\{N(I) \neq 0\}<\infty$ and as always $N(I)$ is almost surely finite. Since the integrand in (12) vanishes for all $N(\cdot)$ in $\Omega$ with $N(I)=0$ it is a simple consequence of dominated convergence that $G\left[\xi_{n}\right] \rightarrow G[\xi]$ as $n \rightarrow \infty$. So by Theorem $4 G[\xi]$ is a p.g.fl, in fact the p.g.fl of the infinitely divisible point process, which proves

THEOREM 5. The p.g.fl of an infinitely divisible point process with KLM measure $\tilde{\mathscr{P}}$ is given by (12). Conversely, if $\tilde{\mathscr{P}}$ has the properties of a KLM measure (12) is the p.g. $f$ of an infinitely divisible point process.

In cases where the p.g.fl is expressible in closed form, (12) gives the associated KLM measure directly (e.g. [13]). We now introduce the class of singular Poisson processes $E_{\lambda, N}$ representing the $n$-fold superposition of point processes independently and identically distributed as $N(\cdot)$ where $n$ is itself a random variable with a Poisson $(\lambda)$ distribution.

Corollary. An infinitely divisible point process has the form $E_{\lambda, N}$ if and only if $\tilde{\mathscr{P}}$ is totally finite. Then $\lambda=\tilde{\mathscr{P}}(\Omega)$ and $N(\cdot)$ has probability measure $\lambda^{-1} \tilde{\mathscr{P}}(\cdot)$.

This result is implicit in Kerstan and Matthes [9]. We note here that the 'curiosity' of Lee ([13] §4) has a totally finite KLM measure and so must be a singular Poisson process. It is in fact a special case of the doubly stochastic Poisson process and (10) shows that doubly stochastic Poisson processes are infinitely divisible if their mean processes have this property. In particular, linear stochastic Poisson processes are all infinitely divisible (Westcott [30]). 


\section{Expansions of the p.g.fi.}

We saw in Section 2 that the most convenient moment measures to use are the factorial moment measures and it turns out they are intimately related to the p.g.fl. Formally, the relationship is simple. If all relevant moment measures exist then on expanding the logarithm and exponential of (6) in their power series we have $([20],[28])$

$$
G[1-\xi]=1+\sum_{k=1}^{\infty} \frac{(-1)^{k}}{k !} \int \cdots \int \xi\left(t_{1}\right) \cdots \xi\left(t_{k}\right) M_{k}\left(d t_{1}, \cdots, d t_{k}\right)
$$

and

$$
H[1-\xi]=\log G[1-\xi]=\sum_{k=1}^{\infty} \frac{(-1)^{k}}{k !} \int \cdots \int \xi\left(t_{1}\right) \cdots \xi\left(t_{k}\right) C_{k}\left(d t_{1}, \cdots, d t_{k}\right) .
$$

To put these relations on a rigorous footing we establish a Taylor-type expansion of the p.g.fl to a finite number of terms, in analogy with results for characteristic functions. This requires

Lemma 5. If $\xi_{1}, \cdots, \xi_{N}$ are real numbers, $0<\xi_{i}<1, i=1, \cdots, N$, where $N$ is an arbitrary positive integer, and we take

$$
\begin{aligned}
Q_{N}=\prod_{i=1}^{N}\left(1-\xi_{i}\right) & =1-\sum_{i=1}^{N} \xi_{i}+\sum_{i_{1}=1}^{N} \sum_{i_{2}>i_{1}} \xi_{i_{1}} \xi_{i_{2}}-\cdots+(-1)^{N} \xi_{1} \cdots \xi_{N} \\
& =1-q_{1}+q_{2}-\cdots+(-1)^{N} q_{N}
\end{aligned}
$$

where

$$
\begin{gathered}
q_{k}=\sum_{1 \leqq i_{1}<\ldots<i_{k} \leqq N} \xi_{i_{1}} \cdots \xi_{i_{k}}, \text { and } \\
S_{N}^{(m)}=1-q_{1}+q_{2}-\cdots+(-1)^{m} q_{m} \quad m=1, \cdots, N,
\end{gathered}
$$

so that $S_{N}^{(m)}$ is the $m$ th partial sum in (15), then

$$
S_{N}^{(2 m-1)} \leqq Q_{N} \leqq S_{N}^{(2 n)}
$$

for all $N$ and $m, n=1,2, \cdots,[N / 2]([x]$ is the greatest integer less than or equal to $x$ ).

Proof. A simple direct proof by induction is possible. However the lemma is a special case of Bonferroni's inequalities (Moran [19] \$1.18) if we interpret the $\xi_{i}$ as the probabilities of a set of independent events $A_{i}$. Putting $m=1$ in Theorem 1.5 of [19], $1-P_{1}$ becomes equal to $Q_{N}$ because of the assumed independence and the lemma follows directly.

$$
\text { COROLIARY. (i) } 0 \leqq Q_{N}-S_{N}^{(2 m-1)} \leqq q_{2 m} \quad 2 m \leqq N
$$



(ii) $0 \leqq Q_{N}-S_{N}^{(2 m-1)} \leqq q_{2 m-1}$
$2 m-1 \leqq N$
(iii) $0 \leqq S_{N}^{(2 m)}-Q_{N} \leqq q_{2 m+1}$
$2 m+1 \leqq N$
(iv) $0 \leqq S_{N}^{(2 m)}-Q_{N} \leqq q_{2 m}$
$2 m \leqq N$

THEOREM 6. For a point process $N(\cdot)$ with p.g. $f G[\xi]$ whose $m$ th factorial moment measure is a Borel measure,

$$
G[1-\rho \xi]=1+\sum_{k=1}^{m} \frac{(-1)^{k}}{k !} \rho^{k} \int \ldots \int \xi\left(t_{1}\right) \cdots \xi\left(t_{k}\right) M_{k}\left(d t_{1}, \cdots, d t_{k}\right)+o\left(\rho^{m}\right),
$$

where $\xi \in V$ and $0<\rho<1$.

Proof. Consider the function

$$
\begin{gathered}
\Gamma_{m}(\rho, \xi)=\rho^{-m}(-1)^{m+1}\left\{\prod_{i}\left[1-\rho \xi\left(t_{i}\right)\right]-1-\sum_{k=1}^{m}(-1)^{k} \frac{\rho^{k}}{k !} \int_{t_{1} \neq \cdot \neq t_{k}} \cdots \int_{k} \xi\left(t_{1}\right) \cdots\right. \\
\left.\cdots \xi\left(t_{k}\right) d N\left(t_{1}\right) \cdots d N\left(t_{k}\right)\right\} .
\end{gathered}
$$

For a realisation $\left\{t_{i}\right\}$ of $N(\cdot)$ we rewrite the integrals as sums so that if $N=N(I)$, $I$ the support of $\xi$,

$$
\Gamma_{m}(\rho, \xi)=\rho^{-m}(-1)^{m+1}\left\{\prod_{i}\left(1-\rho \xi_{i}\right)-1+\rho q_{1}-\rho^{2} q_{2}+\cdots-(-1)^{m} \rho^{m} q_{m}\right\}
$$
where $\xi_{i}=\xi\left(t_{i}\right)$ and the sums are zero for $m>N$.

Since $\xi \in V, N$ is finite with probability one. Because the $m$ th factorial moment measure is a Borel measure, (18) and the Corollary to Lemma 5 show that $\Gamma_{m}(\rho, \xi)$ is positive, bounded by a random variable with finite expectation, namely

$$
(1 / m !) \int_{t_{1} \neq \cdot \neq \int_{t_{m}}} \xi\left(t_{1}\right) \cdots \xi\left(t_{m}\right) d N\left(t_{1}\right) \cdots d N\left(t_{m}\right),
$$

and $\rightarrow 0$ almost surely as $\rho \rightarrow 0$. So by dominated convergence $E\left\{\Gamma_{m}(\rho, \xi)\right\} \rightarrow 0$ as $\rho \rightarrow 0$ for $\xi \in V$, which proves the theorem.

COROLlaRY 1. If the $(m+1)$ st moment measure is also a Borel measure the error term $o\left(\rho^{m}\right)$ is dominated by

$$
\rho^{m+1} \int \cdots \int \xi\left(t_{1}\right) \cdots \xi\left(t_{m+1}\right) M_{m+1}\left(d t_{1}, \cdots, d t_{m+1}\right) .
$$

This follows from (i) and (iii) of the Corollary to Lemma 5. It shows that there is a simple estimate for the remainder term in (17) if we assume the existence of higher-order moment measures.

COROLLARY 2. Under the conditions of the theorem

$$
\mathrm{H}[1-\rho \xi]=\sum_{k=1}^{m} \frac{(-1)^{k} \rho^{k}}{k !} \int \cdots \int \xi\left(t_{1}\right) \cdots \xi\left(t_{k}\right) C_{k}\left(d t_{1}, \cdots, d t_{k}\right)+o\left(\rho^{m}\right) .
$$


Corollary 2 follows from the well-known expression

$$
\log (1-x)=-\left\{x+\frac{x^{2}}{2}+\cdots+\frac{x^{m}}{m}\right\}+o\left(x^{m}\right) .
$$

We remark that here the remainder $o\left(\rho^{m}\right)$ is not estimable in the simple manner of Corollary 1.

A result for characteristic functionals similar to Theorem 6 is given by Shiryaev [26], although he assumes $M_{m+1}(\cdot)$ to exist and has remainder $O\left(\rho^{m+1}\right)$. If we have $1-\xi \in L(N)$ rather than $\xi \in V$ the proof is still valid provided we also assume $\int \cdots \int \xi\left(t_{1}\right) \cdots \xi\left(t_{m}\right) M_{m}\left(d t_{1}, \cdots, d t_{m}\right)<\infty$, which holds automatically when $\xi \in V$ and $M_{m}(\cdot)$ is a Borel measure.

Equation (17) or a direct approach shows that the p.g.fl uniquely determines all existing factorial moment (and cumulant) measures. The method given by Moyal [20] for calculating them directly from the p.g.fl is easily adapted to cover our general situation and we omit the details.

Some limit theorems for point processes are readily established by use of Theorem 6. For example, consider the superposition of $n$ independently and identically distributed stationary orderly point processes $N_{1}(\cdot), \cdots, N_{n}(\cdot)$ with common p.g.fl $G[\xi]$, intensity $\lambda$ and Borel second factorial moment measure $M_{2}(\cdot)$. If we dilate the time scale by a factor $n$ (this is the format of Vere-Jones [28]), the p.g.fl of the superposition is

$$
G_{n}[1-\xi]=(G[1-\xi])^{n}=\left\{1-(\lambda / n) \int \xi(t) d t+R_{n}[\xi]\right\}^{n} \quad \xi \in V
$$

by (17), where by Corollary 1 above

$$
0 \leqq R_{n}[\xi] \leqq \frac{1}{2} M_{2}\left(n^{-1} I \times n^{-1} I\right) .
$$

Here $I$ is the support of $\xi$. But by a theorem of Milne [18] we have

$$
n M_{2}\left(n^{-1} I \times n^{-1} I\right) \rightarrow 0 \text { as } n \rightarrow \infty \text { for all } I,
$$

so (19) gives

$$
\begin{aligned}
G_{n}[1-\xi]= & \left\{1-(\lambda / n) \hat{\xi}(t) d t+o\left(n^{-1}\right)\right\}^{n} \\
& \rightarrow \exp \left\{-\lambda \int \xi(t) d t\right\} \quad \text { as } n \rightarrow \infty .
\end{aligned}
$$

Lemma 4 and (8) now establish that $\sum_{i=1}^{n} N_{i}(\cdot)$ converges to a Poisson $(\lambda)$ process, as is of course well known. There are much weaker forms of this result available (e.g. Grigelionis [4]) but it does illustrate how Theorem 6 provides simple proofs when higher order moments are assumed finite.

\section{Mixing and ergodicity in point processes}

Leonov [14] has given, without proof, a series of theorems characterising ergodicity and mixing in a stationary process in terms of relations involving its 
characteristic functional. We now derive analogous results for point processes and their p.g.fls and give some examples. Similar ideas occur throughout general ergodic theory (e.g. Jacobs [7]).

The concepts of ergodic and mixing point processes were introduced in Section 2. We saw there that both are equivalent to expressions involving limits of probabilities over sets in $\mathscr{F}$ and these are now translated into p.g.fl notation.

THEOREM 7. A stationary point process $N(\cdot)$ with p.g.f $G[\xi]$ is ergodic if and only if

$$
\lim _{t \rightarrow \infty} t^{-1} \int_{0}^{t} G\left[\xi_{1} S^{\boldsymbol{\tau}} \xi_{2}\right] d \tau=G\left[\xi_{1}\right] G\left[\xi_{2}\right]
$$

for $1-\xi_{1}, 1-\xi_{2} \in V$ or $\xi_{1}, \xi_{2} \in L(N)$, where $S^{\tau} \xi(u)=\xi(u-\tau)$

Proof. Suppose $N(\cdot)$ is ergodic so that (1) holds for any $A, B \in \mathscr{F}$ and so certainly for the cylinder sets $\left\{N: N\left(I_{1}\right)=n_{1}, \cdots, N\left(I_{k}\right)=n_{k}\right\}, I_{1}, \cdots, I_{k}$ any Borel sets and $k, n_{1}, \cdots, n_{k}$ any non-negative integers.

Thus

$$
\begin{gathered}
\lim _{\substack{t \rightarrow \infty \\
(21)}} t^{-1} \int_{0}^{t} \mathscr{P}\left\{N\left(I_{1}\right)=n_{1}, \cdots, N\left(I_{k}\right)=n_{k}, N\left(J_{1}+\tau\right)=m_{1}, \cdots, N\left(J_{l}+\tau\right)=m_{l}\right\} d \tau \\
=\mathscr{P}\left\{N\left(I_{1}\right)=n_{1}, \cdots, N\left(I_{k}\right)=n_{k}\right\} \mathscr{P}\left\{N\left(J_{1}\right)=m_{1}, \cdots, N\left(J_{l}\right)=m_{l}\right\}
\end{gathered}
$$

and it follows that the same relation holds for the corresponding p.g.fs, namely

$$
\begin{gathered}
\lim _{t \rightarrow \infty} t^{-1} \int_{0}^{t} P\left(I_{1}, \cdots, I_{k}, J_{1}, \cdots, J_{l} ; y_{1}, \cdots, y_{k}, z_{1}, \cdots, z_{l} ; \tau\right) d \tau \\
=P\left(I_{1}, \cdots, I_{k} ; y_{1}, \cdots, y_{k}\right) P\left(J_{1}, \cdots, J_{l} ; z_{1}, \cdots, z_{l}\right) .
\end{gathered}
$$

Finally since any measurable $\xi$ can be uniformly approximated by an increasing sequence of simple functions, for which (20) holds by (22), we see that (20) is true for arbitrary $\xi_{1}, \xi_{2}$.

Conversely, if (20) holds we deduce (22) on taking $\xi_{1}, \xi_{2}$ as simple functions and hence (21) from the continuity theorem for p.g.fs. So the ergodic relation (1) is established for the cylinder sets of $\mathscr{F}$. That it holds for any measurable sets is a consequence of the following lemma, which is almost certainly known from general ergodic theory. As no specific statement of it has been found we outline the proof.

LEMMA 6. Let $\mathscr{R}$ be a ring, with generated $\sigma$-ring $\mathscr{F}(\mathscr{R})$ and an associated probability space $(\Omega, \mathscr{F}, \mathscr{P})$. Let $T$ be a measure-preserving transformation of $\Omega$ into itself. Then if

$$
\lim _{t \rightarrow \infty} \mathscr{P}\left(A \cap T^{-t} B\right)=\mathscr{P}(A) \mathscr{P}(B)
$$

for all $A, B \in \mathscr{R}$ it holds for all $A, B \in \mathscr{F}(\mathscr{R})$. 
Proof. From Halmos [5] p. 56 we see that for $\varepsilon>0$ and each set $D$ in $\mathscr{F}(\mathscr{R})$ there is a set $D_{\varepsilon}$ in $\mathscr{R}$ with $\mathscr{P}\left(D \Delta D_{\varepsilon}\right)<\varepsilon$. So given $\varepsilon>0$ and arbitrary $A, B$ in $\mathscr{F}(\mathscr{R})$ we find

$$
\begin{aligned}
\left|\mathscr{P}\left(A \cap T^{-t} B\right)-\mathscr{P}\left(A_{\varepsilon} \cap T^{-t} B_{\varepsilon}\right)\right| \leqq & \left|\mathscr{P}\left(A \cap T^{-t} B\right)-\mathscr{P}\left(A_{\varepsilon} \cap T^{-t} B\right)\right| \\
& +\left|\mathscr{P}\left(A_{\varepsilon} \cap T^{-t} B\right)-\mathscr{P}\left(A_{\varepsilon} \cap T^{-t} B_{\varepsilon}\right)\right| \\
& \leqq \mathscr{P}\left(A \Delta A_{\varepsilon}\right)+\mathscr{P}\left(T^{-t} B \Delta T^{-t} B_{\varepsilon}\right) \\
< & 2 \varepsilon \text { for all } t
\end{aligned}
$$

because $T$ is measure-preserving;

$$
\begin{aligned}
\mathscr{P}(A) & \leqq \mathscr{P}\left(A \Delta A_{\varepsilon}\right)+\mathscr{P}\left(A \cap A_{\varepsilon}\right) \\
& <\varepsilon+\mathscr{P}\left(A_{\varepsilon}\right)
\end{aligned}
$$

and similarly for $B$ so that

$$
\left|\mathscr{P}(A) \mathscr{P}(B)-\mathscr{P}\left(A_{\varepsilon}\right) \mathscr{P}\left(B_{\varepsilon}\right)\right|<3 \varepsilon \text { for all } t \text {; }
$$

(iii) $\left|\mathscr{P}\left(A_{\varepsilon} \cap T^{-t} B_{\varepsilon}\right)-\mathscr{P}\left(A_{\varepsilon}\right) \mathscr{P}\left(B_{\varepsilon}\right)\right|<\varepsilon$ for sufficiently large $t$ by hypothesis.

Putting (i), (ii), (iii) together, proof.

$\left|\mathscr{P}\left(A \cap T^{-1} B\right)-\mathscr{P}(A) \mathscr{P}(B)\right|<6 \varepsilon$ for sufficiently large $t$ which completes the

Now the cylinder sets in $\mathscr{F}$ form a semi-ring. Clearly (1) holds for the generated ring and, as an easy consequence of Lemma 6 , it holds for the generated $\sigma$-ring also, that is for $\mathscr{F}$. This establishes the converse proposition and proves Theorem 7 .

An exactly similar argument proves

THEOREM 8. A stationary point process $N(\cdot)$ with p.g. $f$ l $G[\xi]$ is mixing if and only if

$$
\lim _{t \rightarrow \infty} G\left[\xi_{1} S^{t} \xi_{2}\right]=G\left[\xi_{1}\right] G\left[\xi_{2}\right]
$$

for $1-\xi_{1}, 1-\xi_{2} \in \operatorname{Vor} \xi_{1}, \xi_{2} \in L(N)$.

Obviously a like result is true for weakly mixing point processes.

Because the mixing condition (2) or (23) is a form of asymptotic independence of the numbers of points in widely separated intervals we look to these processes for generalisations of the theory of processes with independent increments (i.e. the Poisson process in our field of interest). Similar considerations for mixing sequences of random variables have been studied intensively in recent years.

Since the stationary Poisson process has independent increments it is obviously 
mixing (and hence ergodic), and this can be verified on substituting (8) in (23). Likewise, processes related to the Poisson process should be mixing and we investigate a few examples.

THEOREM 9. A doubly stochastic Poisson process with stationary mean process $\Lambda(t)$ is mixing if $\Lambda$ is mixing.

Proof. We need a slight modification of Leonov's characterisation of mixing stochastic processes. Since $\Lambda(t)$ is non-negative and stationary its Laplace functional is well-defined and from [14] Theorem 1 we easily deduce that $\Lambda$ is mixing if and only if

$$
\lim _{t \rightarrow \infty} L_{\Lambda}\left[\xi_{1}+S^{t} \xi_{2}\right]=L_{\Lambda}\left[\xi_{1}\right] L_{\Lambda}\left[\xi_{2}\right]
$$

for all totally finite measures $\xi_{1}, \xi_{2}$.

Equation (23) is transformed into an expression involving $L_{\Lambda}$ by use of (10). Writing $L[\xi]$ for $L\left[\int_{-\infty}^{t} \xi(u) d u\right]$ we see

$$
G\left[\xi_{1} S^{t} \xi_{2}\right]=\bar{L}_{\Lambda}\left[1-\xi_{1} S^{t} \xi_{2}\right], G\left[\xi_{i}\right]=L_{\Lambda}\left[1-\xi_{i}\right] \quad i=1,2
$$

so that (23) is equivalent to

$$
\lim _{t \rightarrow \infty} L_{\Lambda}\left[1-\xi_{1} S^{t} \xi_{2}\right]=\lim _{t \rightarrow \infty} \bar{L}_{\Lambda}\left[1-\xi_{1}+1-S^{t} \xi_{2}\right] .
$$

Put $\eta_{i}=1-\xi_{i}$, so that $\eta_{i} \in V i=1$,2. Then $1-\xi_{1} S^{t} \xi_{2}=\eta_{1}+\xi_{1} S^{t} \eta_{2}$ and

$$
\begin{aligned}
0 \leqq & \bar{L}_{\Lambda}\left[1-\xi_{1} S^{t} \xi_{2}\right]-\bar{L}_{\Lambda}\left[\eta_{1}+S^{t} \eta_{2}\right] \\
= & E\left\{\left(\exp \left\{-\int \lambda(u)\left[\eta_{1}(u)+\xi_{1}(u) \eta_{2}(u-t)\right] d u\right)\right\}\right. \\
\qquad & \left.\quad\left(1-\exp \left\{-\int \lambda(u) \eta_{1}(u) \eta_{2}(u-t) d u\right\}\right)\right\} \\
& \leqq E\left\{1-\exp \left[-\int \lambda(u) \eta_{1}(u) \eta_{2}(u-t) d u\right]\right\} \\
& \leqq E\left\{\int \lambda(u) \eta_{1}(u) \eta_{2}(u-t) d u\right\} \\
& =E\{\Lambda(u)\} \int \eta_{1}(u) \eta_{2}(u-t) d u
\end{aligned}
$$

since $\Lambda$ is stationary with finite mean. But the $\eta_{i}$ are integrable so the last expression tends to zero as $t \rightarrow \infty$. This establishes (25) and the theorem now follows from Theorem 8.

A corresponding result can be proved if $\Lambda$ is only ergodic. Because linear processes are all mixing (Rosenblatt [23] p. 12) we have 
Corollary. A linear stochastic Poisson process is mixing, and hence ergodic.

From Theorem 7 it is easily shown that a mixed Poisson process is ergodic if and only if its mixing distribution is concentrated at one point, an observation which is clear independently (Theorem 9 does not apply because a process with almost surely constant realisations is obviously not ergodic).

Kerstan and Matthes [10] give a number of results on the mixing and ergodicity of stationary infinitely divisible point processes. Many of them are easily established by p.g.fl methods as follows.

THEOREM 10. A stationary infinitely divisible point process $N(\cdot)$ is mixing if and only if for all bounded intervals $I, J$

$$
\tilde{\mathscr{P}}\{N(I) \neq 0, N(J+\tau) \neq 0\} \rightarrow 0 \text { as } \tau \rightarrow \infty .
$$

Proof. Taking logarithms in (23), mixing is equivalent to

$$
H\left[\xi_{1} S_{2} \xi_{2}\right]-H\left[\xi_{1}\right]-H\left[\xi_{2}\right] \rightarrow 0 \text { as } \tau \rightarrow \infty, 1-\xi_{i} \in V
$$

Applying (12), the left side of (27) becomes

$$
\begin{aligned}
& \int_{\Omega-\{\phi\}}\left\{1-\exp \int \log \xi_{1}(t) d N(t)-\exp \int \log \xi_{2}(t-\tau) d N(t)\right. \\
& \left.\quad+\exp \int \log \xi_{1}(t) \xi_{2}(t-\tau) d N(t)\right\} \tilde{P}(d N) \\
& =\int_{\Omega-\{\phi\}}\left\{1-\exp \int \log \xi_{1}(t) d N(t)\right\}\left\{1-\exp \int \log \xi_{2}(t-\tau) d N(t)\right\} \tilde{P}(d N) .
\end{aligned}
$$

Suppose that $I, J$ are the supports of $1-\xi_{1}, 1-\xi_{2}$ respectively. Then the integrand is zero for the events $\{N(I)=0\}$ and $\{N(J+\tau)=0\}$ so they may be removed from the range of integration. Otherwise, (28) is non-negative and

$$
\begin{aligned}
& \leqq \tilde{\mathscr{P}}\{\Omega-(\{N(I)=0\} \cup\{N(J+\tau)=0\})\} \\
& =\tilde{\mathscr{P}}\{N(I) \neq 0, N(J+\tau) \neq 0\} .
\end{aligned}
$$

If this converges to zero as $\tau \rightarrow \infty$ for any bounded $I, J(27)$ is true and the process is mixing. Conversely, if the process is mixing then by choosing $\xi_{1}, \xi_{2}$ to be zero over $I, J$ respectively we see from (27) and (28) that (26) holds which completes the proof.

COROllary. Regular infinitely divisible point processes are all mixing.

This follows directly from the definition of such processes in Section 2. In fact, as the regular processes are all Poisson cluster processes (Matthes [16]) the corollary is also a consequence of some general results on mixing in cluster processes proved by Wescott [31]. 
The method used to prove Theorem 10 is readily adapted to the proof of other propositions of Kerstan and Matthes, such as that for stationary infinitely divisible point processes weak mixing is equivalent to ergodicity.

\section{Additional remarks}

The pertinent question of why we choose to work with the p.g.fl rather than the characteristic functional (Bartlett [2]) can now be answered. In the first place we saw that the most useful moment measures for a point process are the factorial moment measures, and these are generated by the p.g.fl. Secondly, Lemma 4 suggests the possibility of constructing a general theory of limit theorems for point processes in terms of convergence of some class of associated functionals. Most such theorems assert convergence to the Poisson process and it is clear from (7) or (8) that this is most naturally associated with the logarithm of the p.g.fl. We remark however that such a theory has not yet eventuated, though several specific problems have been successfully tackled, and this is certainly an area worth further investigation. One approach would be to find a workable bound to the error term in Corollary 2 of Theorem 6.

Another area in which the p.g.fl has proved useful is the study of cluster point processes (e.g. [20], [29]), since the p.g.fl of such processes has a pleasantly compact formulation. It is intended to publish applications of our approach to questions of existence and mixing for cluster processes in the near future.

The author is grateful to Professor D. Vere-Jones for his continued guidance, to Professor P. A. Moran for suggesting the proof of Lemma 5 and Dr M. Jiřina for assistance with the material of Section 6 .

A comprehensive survery of the theory of point processes has been given by Paley and Vere-Jones [32].

\section{References}

[1] M. S. Bartlett, 'The spectral analysis of point processes', J. Roy. Statist. Soc. Ser. B. 25 (1963), 264-296.

[2] M. S. Bartlett, Stochastic Processes (C. U. P., 2nd ed. 1966).

[3] D. R. Cox and P. A. W. Lewis, The Statistical Analysis of Series of Events (Methuen, 1966).

[4] B. Grigelionis, 'On the convergence of random step processes to a Poisson process', Th. Probab. Appl. 8 (1963), 177-182.

[5] P. R. Halmos, Measure Theory (Van Nostrand, 1950).

[6] T. E. Harris, The Theory of Branching Processes (Springer, 1963).

[7] K. Jacobs, 'On Poincaré's recurrence theorem', Proc. 5th Berkeley Symp. 2, II (1965), 375-404.

[8] M. Jiř ina, 'Asymptotic behaviour of measure-valued branching processes', Rozpravy Českosl. Akad. Věd. R. Mat. Privodnich Věd. 76, 3 (1966).

[9] J. Kerstan and K. Matthes, 'Stationä rezufällige Punktfolgen II', J-ber. Deutsch. Math. Verein. 66 (1963), 106-118.

[10] J. Kerstan and K. Matthes, 'Ergodische unbegrenzt teilbare stationäre zufällige Punktfolgen', Trans. 4th Prague Conf. (1965), 399-415. 
[11] A. Ya. Khintchine, Mathematical Methods in the Theory of Queueing (Griffin, 2nd ed., 1969).

[12] P. M. Lee, 'Infinitely divisible stochastic processes', $Z$. Wahrscheinlichkeitstheorie verw. Geb. 7 (1967), 147-160.

[13] P. M. Lee, 'Some examples of infinitely divisible point processes', Stud. Sci. Math. Hung. 3 (1968), 219-224.

[14] V. P. Leonov, "Applications of the characteristic functional and semi-invariants to the ergodic theory of stationary processes', Dokl. Acad. Nauk SSSR (1960), 523-526.

[15] K. Matthes, 'Stationäre zufällige Puntkfolgen I', J-ber. Deutsch Math. Verein. 66 (1963), 66-79.

[16] K. Matthes, 'Unbeschränkt teilbare Vesteilungsgesetze stationärer zufälliger Punktfolgen', Wiss. Z. Hochschule Elektro Ilmenau 9 (1963), 225-238.

[17] J. Mecke, 'Stationäre zufällige Masse auf Lokalkompakten Abelschen Gruppen', Z. Wahrscheinlichkeitstheorie verw. Geb. 9 (1967), 36-58.

[18] R. K. Milne, 'Simple proofs of some theorems on point processes', Ann. Math. Statist. 42 (1971) 368372.

[19] P. A. P. Moran, An Introduction to Probability Theory (O. U. P., 1968).

[20] J. E. Moyal, 'The general theory of stochastic population processes', Acta Math. 108 (1962), 1-31.

[21] J. E. Moyal, 'Multiplicative population chains', Proc. Roy. Soc. A 266 (1962), 518-526.

[22] K. Nawrotzki, 'Eine Grenzwertsatz für homogene zufällige Punktfolgen', Math. Nachr. 24 (1962), 201-217.

[23] M. Rosenblatt, Random Processes (O. U. P. 1962).

[24] C. Ryll-Nardzewski, 'Remarks on the Poisson stochastic process (III)', Studia. Math. 14 (1954), 314-318.

[25] C. Ryll-Nardzewski, 'Remarks on processes of calls', Proc. 4th Berkeley Symp. 2 (1960), 455-465.

[26] A. N. Shiryaev, 'Some problems in the spectral theory of higher order moments I', Th. Probab. Appl. 5 (1960), 265-284.

[27] I. M. Slivnyak, 'Some properties of stationary flows of homogeneous random events', Th. Probab. Appl. 7 (1962), 336-341 (see also 9, (1964), 168).

[28] D. Vere-Jones, 'Some applications of probability generating functionals to the study of inputoutput streams', J. Roy. Statist. Soc. Ser. B 30 (1968), 321-333.

[29] D. Vere-Jones, 'Stochastic models for earthquake occurrence', J. Roy. Statist. Soc. Ser. B $32(1970), 1-62$.

[30] M. Westcott, 'Identifiability in linear processes', Z. Wahrscheinlichkeistheorie verw. Geb. 16 (1970), 39-46.

[31] M. Wescott,. 'On existence and mixing results for cluster point processe', J. Roy. Statist. Soc. Ser. B. 33 (1971), 290-300.

[32] D. J. Daley and D. Vere-Jones, 'A summary of the theory of point processes', in Stochastic Point Processes: Statistical Analysis, Theory and Application, edited by P. A. W. Lewis 299-383 (Wley, 1972).

Department of Statistics

Australian National University

Canberra

Present address,

Department of Mathematics

Imperial College of Science and Technology

London SW7 2PJ 